\title{
Funcionamento Familiar: Revisão de Estudos Empíricos sobre Medidas de Avaliação (FAD, FACES-IV e SCORE-15)
}

\author{
Family Functioning: A Review of Empirical Studies on Assessment Measures (FAD, \\ FACES-IV and SCORE-15)
}

\author{
Marisa Santos ${ }^{1}$, Margarida Vilaça ${ }^{2}$, Alda Portugal $^{3}$ e Ana Paula Relvas ${ }^{4}$
}

\begin{abstract}
Resumo
As teorias psicológicas sobre a família definem fatores que devem ser avaliados como a composição e o processo familiar, os quais incluem interações, afetividade, papéis e regras dos membros da família. Este estudo visa a análise dos instrumentos de avaliação familiar frequentemente utilizados na Europa e no Brasil nos últimos 11 anos. Selecionaram-se artigos de pesquisas empíricas, sendo que a maioria dos resultados validou os instrumentos Family Assessment Device (FAD), Family Adaptability and Cohesion Evaluation Scales IV (FACES-IV) e Systemic Clinical Outcome and Routine Evaluation-15 (SCORE-15). A escala do FAD General Functioning of Family (GFF) e o SCORE-15 obtiveram melhores avaliações em pesquisas transculturais. O SCORE-15 destaca-se pelos resultados mais promissores: trata-se de um medida familiar contemporânea e breve, com sensibilidade à mudança terapêutica comprovada, e praticidade na aplicação.
\end{abstract}

Palavras-chave: FAD, FACES-IV, SCORE-15, funcionamento familiar

\begin{abstract}
Psychological theories regarding family functioning suggest the assessment of specific variables, such as family process and its composition, which includes dimensions as interactions, affection, rules and roles of family members. The present study aims to analyze family measures widely used to assess family functioning in Brazil and Europe in the past 11 years. Empirical research, selected according to specific criteria, reveals that the Family Assessment Device (FAD), Family Adaptability and Cohesion Evaluation Scales IV (FACES-IV) and Systemic Clinical Outcome and Routine Evaluation-15 (SCORE-15) represent valid assessment tools. General Functioning of Family (GFF), a FAD subscale, and SCORE-15 obtained more adequate psychometric properties in transcultural studies. SCORE-15 stands out due to its promising results: it is a brief contemporary family measure, with proven sensitiveness to therapeutic change and ease of use.
\end{abstract}

Keywords: FAD, FACES-IV, SCORE-15, family functioning

\footnotetext{
${ }^{1}$ Mestre em Psicologia Clínica pela Pontifícia Universidade Católica de Campinas, Brasil. Doutoranda da Faculdade de Psicologia e de Ciências da Educação da Universidade de Coimbra, Portugal. Rua Vila Pedro Lima, n. 65 cs 08- Macaé-RJ.CEP 27930780 Tel. +55 22 997496817. E-mail: marisaalves2003@yahoo.com.br

${ }^{2} \mathrm{PhD}$ em Psicologia Clínica. Investigadora, Centro de Investigação em Psicologia, Escola de Psicologia da Universidade do Minho, Braga. Campus de Gualtar, 4710-057, Braga, Portugal. Tel.: 916070964. E-mail: margaridavilaca@psi.uminho.pt

${ }^{3} \mathrm{PhD}$ em Psicologia Clínica. Professora, Departamento de Psicologia da Universidade da Madeira, Madeira, Portugal. Centro de Estudos Sociais da Universidade de Coimbra, Coimbra, Portugal. Campus Universitário da Penteada, 9020-105 Funchal, Portugal. Tel.: 915894872. E-mail: alda.portugal@staff.uma.pt

${ }^{4} \mathrm{PhD}$ em Psicologia Clínica. Professora catedrática, Faculdade de Psicologia e de Ciências da Educação da Universidade de Coimbra, Investigadora do Centro de Estudos Sociais da Universidade de Coimbra, Coimbra. Rua do Colégio Novo 3000-115 Coimbra, Portugal. Tel.: +351239851450. E-mail: aprelvas@fpce.uc.pt
}

Revista Iberoamericana de Diagnóstico y Evaluación - e Avaliação Psicológica. RIDEP · No61 · Vol.4 · 49-64 · 2021

ISSN: 1135-3848 print /2183-6051online 


\section{Introdução}

\section{Definição de funcionamento familiar e relevância do seu estudo e avaliação}

A definição do conceito de "funcionamento familiar" nem sempre é consensual na literatura, dada a diversidade de teorias e diferentes perspectivas sobre o tema. Bray (1995) considera que existe dificuldade na definição do funcionamento familiar saudável e do funcionamento familiar patológico. Dessa forma, o autor sugere que sejam analisadas quatro grandes categorias com vista a analisar $\mathrm{o}$ funcionamento familiar, designadamente: 1) composição familiar, isto é, a descrição da estrutura da família e dos elementos que a compõem; 2) processo familiar, ou seja, os comportamentos e interações que caracterizam as relações familiares, tais como conflito, diferenciação, comunicação, resolução de problemas e controle; 3) fatores afetivos, como emoções e expressão afetiva entre os elementos da família; e 4) organização familiar, que diz respeito aos papéis e às regras na família, incluindo aspectos como limites/fronteiras e hierarquia (Bray, 1995).

De acordo com Minuchin (1982), pioneiro da terapia estrutural, tanto os limites geracionais claros, como a definição clara de papéis e funções familiares, constituem critérios importantes para determinar se o funcionamento familiar é ou não saudável. Estudar o funcionamento familiar e a sua complexidade afigura-se uma tarefa muito relevante e atual, especialmente quando se consideram as transformações sociais e culturais ocorridas nas últimas décadas que facilitaram a emergência de novas formas de família (Dessen, 2010; Teodoro, 2012; Walsh, 2016). Para o efeito, a teoria sistémica e os diferentes instrumentos terapêuticos e de medida auxiliam o profissional que se dedica ao trabalho com famílias e casais, bem como o pesquisador na busca de respostas acerca dos processos familiares atuais, que se caracterizam pelo dinamismo, instabilidade e tendência transformadora nas relações familiares. Ao longo dos últimos anos, vários estudos desenvolvidos com população comunitária (Baiocco et al. 2013; Koutra et al., 2012) e clínica (O'Hanrahan et al., 2017; Paolini \& Schepisi, 2020; Stratton et al., 2014) têm evidenciado a importância da compreensão do funcionamento familiar, considerando a perspetiva de todos os elementos do sistema familiar, uma vez que esta potencia a eficácia das intervenções familiares. No entanto, e apesar de existir evidência empírica sobre a associação entre o funcionamento familiar e a adaptação psicossocial das famílias e dos seus membros (e.g., Ballash et al., 2006; Van Schoors et al., 2016), são escassos os instrumentos de medida disponíveis e devidamente validados com vista a avaliar o funcionamento familiar, particularmente no contexto Brasileiro (e.g., Teodoro, 2012).

Lebow e Stroud (2016) questionam as medidas existentes alegando que os pesquisadores tomaram como critério de normalidade apenas as famílias que participam nas pesquisas, geralmente formadas por pais e filhos brancos de classe média. Atualmente, com o crescente número de divórcios, recasamentos e famílias monoparentais, há que pensar em medidas adequadas às novas configurações, que apresentem novas questões a serem respondidas pelo clínico e pelo pesquisador. Pesquisas recentes desenvolvidas em contexto clínico (Hamilton et al., 2015; Jósefik et al., 2016; Paolini \& Schepisi, 2020; Stratton et al., 2014) reforçam a relevância da avaliação familiar ao longo do processo terapêutico como forma de monitorar a evolução da terapia. Da mesma forma, Staccini et al. (2015), após uma revisão de estudos, vêm enfatizar a importância da introdução da mensuração da dinâmica familiar do paciente na avaliação da clínica médica. Estudos de meta-análise (Carr, 2009a, 2009b) confirmaram que as intervenções sistémicas são eficazes não só em adultos, mas também em crianças e adolescentes com problemas de saúde mental, acrescentando que a terapia familiar sistémica se mostra adequada à resolução de problemas infantis e juvenis por envolver todo o sistema familiar. Nesse sentido, a avaliação e a intervenção terapêutica ao nível da família podem ser um complemento eficaz na melhoria da saúde física e mental global.

\section{Avaliação do funcionamento familiar no Brasil}

Como referido anteriormente, verifica-se uma escassez de instrumentos de avaliação sobre o funcionamento familiar no contexto Brasileiro (e.g., Teodoro, 2012), pese embora exista 
evidência empírica clara sobre a associação entre esta dimensão e o funcionamento dos indivíduos. Neste sentido, os Cadernos de Atenção Básica Saúde Mental (Ministério da Saúde, 2013) reforçam a importância da abordagem familiar pelos profissionais de saúde em geral, pontuando a visão sistémica na sua atuação no Sistema Único de Saúde Brasileiro. Souza et al. (2011) realizaram uma revisão sistemática sobre a avaliação familiar no contexto da saúde mental no Brasil, tendo sido encontradas poucas escalas validadas no país. Os autores concluíram, ainda, que a melhoria do funcionamento familiar parece estar associada à recuperação do paciente com transtorno mental, pontuando que a avaliação familiar poderá ser uma metodologia adequada para a identificação dos fatores do ambiente familiar que podem auxiliar no planejamento terapêutico e na reabilitação do paciente psiquiátrico. Pavarini et al. (2020) analisaram os preditores do funcionamento familiar em idosos cuidadores de cônjugues idosos, revelando que a presença de stresse, depressão, declínio cognitivo e número de filhos são preditores do funcionamento familiar e devem ser considerados no contexto da saúde geral do idoso. Num outro estudo, Ferriolli et al. (2007) mostraram como variáveis do contexto familiar (e.g., stresse materno, instabilidade financeira) se associam a problemas de saúde mental da criança. PereiraSilva et al. (2015) em pesquisas com casais com e sem filhos com deficiência intelectual revelaram que a deficiência da criança não parece constituir um obstáculo no ajustamento do casal, fato que deveria ser melhor investigado em famílias brasileiras, segundo os autores.

No Brasil, mesmo não havendo tradição em avaliação do funcionamento familiar, a literatura e o crescimento da terapia familiar já vêm se consolidando por décadas. Teodoro (2012) pontua que há dificuldade de sistematização de instrumentos de avaliação familiar a partir da própria definição de família pelos pesquisadores brasileiros, que segue o modelo internacional de família nuclear, ou seja, a formada por pais e filhos, limitando-se ao estudo de relações entre os citados membros.

O Conselho Federal de Psicologia é a autarquia responsável pela aprovação de instrumentos psicológicos (Resolução n. 9, de 25 de abril de 2018). Entre aqueles aprovados e direcionados à avaliação familiar, destacam-se os Inventários de Habilidades Sociais (versões para o pais, adolescentes e crianças) e o Inventário de Percepção de Suporte Familiar (Conselho Federal de Psicologia, n.d.).

Considerando-se, então, a falta de instrumentos de autorrelato que abordem dimensões gerais e essenciais ao funcionamento familiar, validados para a população brasileira, e, consequentemente, a carência de pesquisas empíricas focadas no funcionamento familiar no Brasil, o presente estudo tem como objetivos: (1) fazer um levantamento dos instrumentos de avaliação familiar mais utilizados no Brasil e internacionalmente; e (2) analisar de forma detalhada as características dos instrumentos identificados no objetivo anterior, particularmente em termos de constructo(s) teórico(s) avaliado(s), características psicométricas, populações e contextos de aplicação. Desta forma, pretende-se que o estudo aqui proposto contribua para colmatar a lacuna existente ao nível da avaliação familiar e do estudo empírico da dinâmica familiar, no contexto brasileiro.

\section{Método}

Com o objetivo de identificar os instrumentos de avaliação do funcionamento familiar mais utilizados no Brasil, mas também internacionalmente, $\mathrm{e}$ analisar as respetivas características, procedeu-se a uma pesquisa em diferentes bases de dados de literatura científica, especificamente na B-on, Academic Search, Scopus, Pro-Quest, Scielo e Google Acadêmico. Os critérios de inclusão foram: serem (1) estudos empíricos (2) publicados na última década, especificamente entre 2009 e 2020, (3) em inglês, português e espanhol, e (4) que se reportassem a estudos de validação (ou que incluíssem dados de validade psicométrica) de instrumentos de autorrelato sobre o funcionamento familiar. A recolha da amostra foi efetuada em dois passos sequenciais.

No primeiro momento, de cariz exploratório, as palavras-chave utilizadas na pesquisa foram: family functioning, family assessment, validation, family relations e family communication. Nesta altura, os artigos encontrados foram triados a partir 


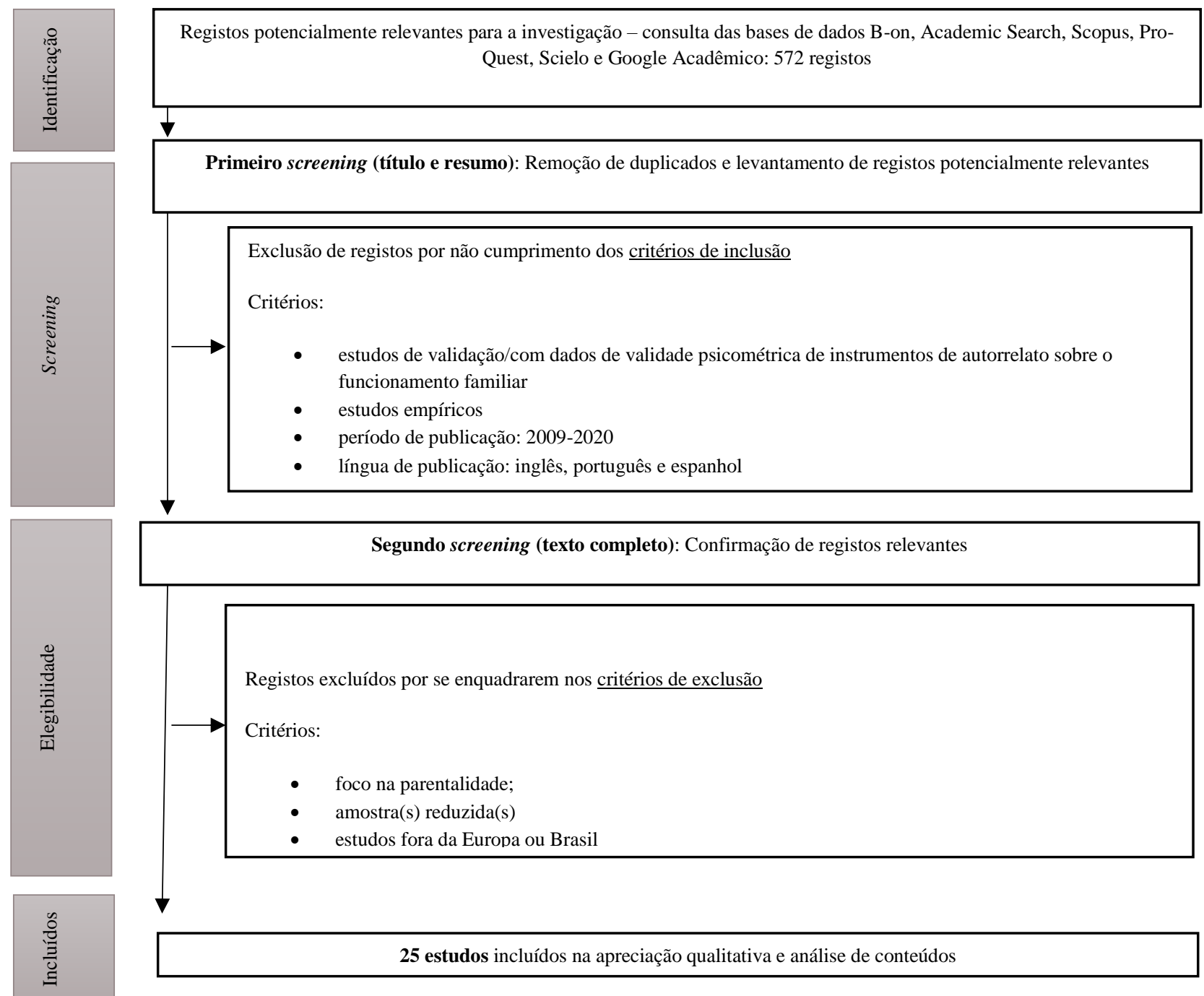

Figura 1. Fluxograma de seleção dos artigos sobre medidas de avaliação do funcionamento familiar: FAD, FACES-IV e SCORE-15 (pós pesquisa exploratória)

Nota. FACES-IV=Family Adaptability and Cohesion Evaluation Scale-IV; FAD=Family Assessment Device; GFF=General Functioning of Family; SCORE-15=Systemic Clinical Outcome and Routine Evaluation-15.

da leitura dos sumários, considerando os critérios de inclusão; os artigos selecionados foram, então, avaliados em texto completo. Concluída esta fase introdutória da pesquisa, os autores concluíram que os instrumentos mais frequentemente referidos eram o Family Assessment Device (FAD; Epstein et al., 1983) e a sua subescala General Family Functioning (GFF), que é utilizada de forma independente em diversos estudos; a Family Adaptability and Cohesion Evaluation Scale IV (FACES-IV; Olson, 2000) e o Systemic Clinical Outcome and Routine Evaluation-15 (SCORE-15; Stratton et al., 2010). Esta análise inicial permitiu concluir ainda que a grande maioria dos estudos sobre medidas de avaliação familiar eram oriundos da Europa. Desta forma, a partir deste momento, consideraram-se apenas os estudos desenvolvidos no Brasil e na Europa.
Avançou-se então para o segundo momento da pesquisa, focado na análise dos instrumentos evidenciados na fase anterior. Para além dos critérios de inclusão considerados no primeiro momento, acrescentaram-se os seguintes critérios de exclusão: (1) estudos com foco na parentalidade; (2) pesquisas empíricas com uma amostra com população $(N)$ reduzida; (3) conduzidos em áreas geográficas que não a Europa e/ou Brasil; e (4) estudos focados noutros instrumentos de avaliação que não o FAD/GFF, a FACES-IV e o SCORE-15. Refinou-se o foco da pesquisa, acrescentando às palavras-chave o nome dos instrumentos que emergiram mais frequentemente na análise do primeiro passo: $\mathrm{fad}$, family assessment device, general family functioning subscale, family adaptability cohesion evaluation scales, faces iv, systemic clinical 
outcome and routine evaluation e score-15. No Scielo Brasil foram utilizadas, complementarmente, as palavras funcionamento familiar e adaptação transcultural a fim de otimizar a pesquisa de artigos publicados no Brasil e em português. Neste segundo passo, os artigos encontrados foram sujeitos aos procedimentos de seleção idênticos aos utilizados no primeiro.

\section{Resultados}

De um total de 572 artigos inicialmente levantados, foram identificados como cumprindo os critérios de inclusão e exclusão 25 artigos, distribuídos desta forma: oito artigos sobre o SCORE-15, oito artigos sobre a FACES-IV e nove artigos sobre o FAD/GFF. Esses se encontram sistematizados nos Quadros 1, 2 e 3.

\section{Family Assessment Device}

O FAD (Epstein et al., 1983) é um instrumento de autorresposta desenvolvido com o objetivo de identificar áreas problemáticas do funcionamento familiar. Suportado teoricamente no Modelo McMaster do Funcionamento Familiar (Epstein et al., 1983), essa medida de avaliação parte de alguns pressupostos: 1) todos os membros da família estão interconectados, isto é, uma parte da família não pode ser compreendida isoladamente do resto do sistema familiar; 2) o funcionamento familiar não pode ser compreendido apenas pelo comportamento individual de seus membros; 3) a estrutura e a organização familiares são fatores que influenciam e determinam o comportamento dos membros da família; e 4), por último, os padrões transacionais do sistema familiar modelam o comportamento de seus membros.

$\mathrm{O}$ FAD, tendo em consideração esses pressupostos, debruça-se sobre a avaliação do funcionamento familiar, sendo constituído por 60 itens distribuídos por sete dimensões da vida familiar: Resolução de problemas (e.g., "Resolvemos a maioria dos conflitos emocionais que surgem"), Comunicação (e.g., "Somos francos uns com os outros"), Papéis familiares (e.g., "As tarefas familiares não são distribuídas de maneira igual"), Responsividade afetiva (e.g., "Expressamos nosso carinho"), Envolvimento afetivo (e.g., "Se alguém está com problemas, os outros se envolvem muito"), Controle do comportamento (e.g., "Não obedecemos às regras ou normas") e Funcionamento geral familiar (e.g. "É difícil planejar atividades familiares porque não nos entendemos") (Epstein et al., 1983; Miller et al., 2000). Essa última dimensão, descrita na literatura como GFF, é frequentemente utilizada de modo independente relativamente à escala total, dado que os 12 itens que a compõem representam, de forma significativa, o funcionamento geral da família. Tanto o FAD quanto a GFF podem ser administrados a todos os membros da família com idade superior a 12 anos, pretendendo-se que o respondente analise cada afirmação de acordo com quatro alternativas de intensidade: 1=Concorda Muito; 2=Concorda; 3=Discorda; e 4=Discorda Muito. O preenchimento do FAD demora, em média, de 15 a 20 minutos e é útil no contexto clínico; a GFF, por sua vez, pode ser utilizada como instrumento de triagem, uma vez que os 12 itens que a compõem tendem refletir de forma geral e fiel o funcionamento familiar. Seguidamente, o Quadro 1 sistematiza algumas das pesquisas realizadas com o FAD e/ou com a subescala GFF.

A análise dos estudos apresentados no Quadro 1 mostrou que a maioria realizou validação em diferentes países europeus, seja na versão original do FAD, seja na versão GFF e, também, na versão na proposta por Timmerby et al. (2018) de 36 itens. Observou-se uma tendência de o instrumento ser mais bem avaliado na versão GFF (Almeida et al., 2020; Pires et al., 2016). As pesquisas de adaptação cultural do FAD (Barroilhet et al., 2009; Kazarian, 2010; Speranza et al., 2012) pontuaram a necessidade de revisão de subscalas como a Affective responsiveness (Responsividade afetiva) e a Affective Involvement (Envolvimento afetivo), as quais avaliam a dinâmica emocional familiar. A versão espanhola de Barroilhet et al. (2009) revela que essa dinâmica emocional parece ter maior peso e valor para os membros da família nas culturas hispânicas do que em outras culturas (Padilla, 2002). García-Méndez et al. (2006) também corroboram a citada afirmação, enfatizando que a família é central na vida dos mexicanos.

Quanto às amostras da população avaliada houve uma equiparação entre mistas, clínica e comunitária, com predominância de participantes 
Quadro 1. Sistematização de pesquisas realizadas com o FAD e a sua subescala GFF

\begin{tabular}{|c|c|c|c|}
\hline Referência - país & Objetivos & População / $n$ & Resultados \\
\hline $\begin{array}{l}\text { Almeida et al. } \\
(2020)- \\
\text { Portugal }\end{array}$ & $\begin{array}{l}\text { Analisar as PP da GFF em } \\
\text { famílias com adolescentes } \\
\text { diabéticos tipo I comparando } \\
\text { com adolescentes saudáveis. }\end{array}$ & $\begin{array}{l}100 \text { famílias portuguesas (pais) } \\
\text { com adolescentes diabéticos de } \\
\text { tipo I e } 106 \text { famílias (pais) com } \\
\text { adolescentes saudáveis. }\end{array}$ & $\begin{array}{l}\text { Revelaram-se boas PP e confiabilidade em famílias com } \\
\text { adolescentes diabéticos e saudáveis. A AFC de ambas amostras } \\
\text { indica um modelo unifatorial com bons índices de ajustamento. } \\
\text { Obteve-se boa consistência interna ( } \alpha \text { entre } .84 \text { e } .79 \text { ), validade } \\
\text { divergente e convergente nos dois grupos. }\end{array}$ \\
\hline
\end{tabular}

Timmerby et al.

(2018) -

Dinamarca

Analisar as PP da versão breve da FAD (36 itens)

Descrever o processo de

Pires et al. (2016) - Brasil adaptação transcultural da GFF e analisar as PP do instrumento.

Tradução e adaptação transcultural da FAD

Traebert et al. (português do Brasil) e análise da consistência interna.

564 pacientes adultos com cancro e membros de suas famílias, de diferentes nacionalidades (Suíça, Alemanha, Reino Unido, Finlândia, Áustria e Dinamarca).

A ACP revelou uma estrutura bifatorial (18 itens avaliam Bemestar familiar e 18 itens avaliam Disfuncionamento familiar). Não foi estabelecido ponto de corte para a população clínica.

Estudo de revisão sistemática qualitativa para identificação das propriedades

Staccini et al. clinicométricas

(2015) - Itália

(sensibilidade $\mathrm{e}$ responsividade) do FAD e reportar sua utilização no contexto clínico.

Banovcinova e Avaliar a relação entre o Levicka (2015) - rendimento familiar e a Eslováquia comunicação na família.

Speranza et al. Validação da versão francesa (2012) - França do FAD

Kazarian (2010) - Líbano

Adaptação cultural da versão arménia da FAD de 60 itens.
500 pais e mães de crianças (6 a 8 anos) do $2^{\mathrm{a}}$ ano do ensino fundamental brasileiro.

80 adultos com idade média de 33.9 anos.

558 adolescentes armênios com idade média de 16 anos.

Equivalência semântica na ordem dos $90 \%$ e $100 \%$. Valores de consistência interna $(\alpha)$ entre .79 e .81 . A AFC revelou uma escala unidimensional. $\mathrm{O}$ instrumento demonstrou validade de constructo (mães e pais que bebem mais álcool apresentam pior FF)

Durante o processo de tradução verificou-se a necessidade de rever algumas palavras/itens. A consistência interna $(\alpha)$ para a amostra total foi de .91 (resultados por dimensão variam entre $.91 \mathrm{e} .86)$.

Na maioria dos estudos, o FAD foi capaz de discriminar entre população clínica e de controle, e entre grupos de pacientes ou de diferentes doenças. O FAD apresentou ainda bons indicadores de estabilidade temporal, assim como modesta sensibilidade para mudanças terapêuticas após intervenção.

310 participantes de nível socioeconómico baixo; 310 participantes de nível socioeconómico médio.

323 participantes: 115 do grupo não clínico (grupo 1), 102 familiares de pacientes psiquiátricos (grupo 2) e 106 pacientes clínicos e suas famílias (grupo 3).

240 indivíduos: 60 pacientes psiquiátricos e suas famílias; e 60 participantes e suas famílias em grupo de controle.
$59.7 \%$ das famílias com rendimentos abaixo da média revelaram pior comunicação familiar. Por outro lado, $47.7 \%$ das famílias com rendimentos médios revelaram comunicação familiar problemática.

A ACP revelou uma estrutura tridimensional. Obteve-se boa estabilidade temporal (com 20 participantes da amostra não clínica; .78 e .90). Encontraram-se valores de consistência interna $(\alpha)$ moderados a baixos, à exceção da escala GF (grupo 1: .82; grupo 2: .48; grupo 3: .64). A validade discriminante revelou que o grupo psiquiátrico obteve valores consideravelmente mais elevados do que os outros grupos $(p<.05)$.

Boa consistência interna global $(\alpha=.89)$, porém, valores menos satisfatórios nas 6 dimensões, comparativamente ao estudo original. As correlações elevadas com outras medidas de avaliação da coesão familiar confirmaram a validade convergente $(r=-0.55 ; p<.0001)$. Verifica-se validade que os rapazes $(p<.01)]$

A consistência interna para a amostra total foi de $\alpha=.94$ Obteve-se uma estabilidade temporal adequada (entre .77 e .91 ) e validade discriminante significativa $(p<.001)$ em todas as subescalas do FAD. A análise fatorial sugere um modelo trifatorial, distinto do modelo original. discriminante para o género [as jovens reportam melhor FF do

$\begin{array}{ll}\text { Barroilhet et al. } & \text { Analisar as PP da versão } \\ \text { (2009) - } & \text { espanhola do FAD. }\end{array}$

Nota. $\mathrm{ACP}=$ análise de componentes principais; $\mathrm{AFC}=$ análise fatorial confirmatória; $\mathrm{FAD}=$ Family Assessment Device; $\mathrm{FF}=$ funcionamento familiar; GFF=General Functioning of Family; $\mathrm{PP}=$ propriedades psicométricas.

adultos. Os temas de estudo também mostraramse equipados em números e variedade, apresentando estudos com doenças clínicas (cancro e diabetes tipo I), pacientes de saúde mental e problemas psicossociais (violência familiar e diferenças socioeconômicas). As informações sobre a amostra (clínica e comunitária) revelam a preocupação dos pesquisadores em obter maior representatividade, diversificando os grupos analisados a fim de almejar melhores resultados psicométricos. Por outro lado, a predominância da população adulta limita os resultados porque não tem a percepção de outros elementos da família que se encontram em diferentes ciclos de vida familiar.

Os temas estudados contribuíram, também, com conhecimentos acerca de pacientes psiquiátricos, pacientes clínicos com doenças crónicas e problemas comportamentais graves como a violência intrafamiliar e dificuldade de 
comunicação nas famílias com baixo nível econômico. Quanto às propriedades psicométricas, a maioria das pesquisas obtiveram boa consistência interna e estabilidade temporal. Duas pesquisas (Pires et al., 2016; Timmerby et al., 2018) pontuaram a necessidade de ponto de corte.

Em síntese, constata-se que a utilização do FAD, mais especificamente da GFF, apresenta confiabilidade e validação nas pesquisas empíricas realizadas em diferentes países europeus, apresentando, globalmente, boas propriedades psicométricas. Observa-se, também, que o FAD se revelou útil no setting clínico, auxiliando na avaliação médica em relação a várias doenças. Convém ressaltar que as pesquisas transculturais do FAD versão original revelaram resultados diferentes em certas dimensões, apontando, assim, a necessidade de novos estudos culturais.

\section{Family Adaptability Cohesion Evaluation Scale-IV}

A FACES-IV é uma escala de avaliação familiar que se ancora no Modelo Circumplexo dos Sistemas Conjugal e Familiar proposto por Olson (2000). A hipótese central sugere que níveis equilibrados de coesão e flexibilidade levam a um funcionamento familiar mais saudável, enquanto níveis desequilibrados (muito baixos ou muito altos) estão associados a um funcionamento familiar problemático. A FACES-IV foi construída ao longo de vários anos por Olson e colaboradores (Olson, 2000; 2011; Olson et al., 1979; Olson \& Gorall, 2003; Olson et al. 2007) e possui vários estudos de validação em países da Europa, América do Sul e Estados Unidos, o que permitiu o seu aperfeiçoamento até a última versão (Olson, 2011). Esta versão é um instrumento de 42 itens que mede o funcionamento familiar a partir de três dimensões: 1) Coesão, que diz respeito à ligação emocional estabelecida entre os elementos de uma família e com outros; 2) Flexibilidade, que reflete a capacidade da família para alterar sua estrutura de poder, bem como as regras, papéis e relações entre os seus elementos no sentido de ser capaz de dar resposta a situações de crise e 3) Comunicação, considerada uma dimensão facilitadora das interações e do equilíbrio familiar, uma vez que, quando positiva, permite ao casal e à família como um todo ajustarem seus níveis de coesão e de flexibilidade. Os 42 itens são distribuídos por seis dimensões específicas (cada uma contém sete itens) que se encontram agrupadas em dois fatores de segunda ordem, coesão e flexibilidade, designadamente: Coesão emaranhada (e.g., "Passamos muito tempo juntos"), Coesão balanceada (e.g., "Os membros da família envolvem-se na vida uns dos outros"), Coesão desengajada (e.g., "Damo-nos melhor com pessoas de fora da família do que entre nós"), Flexibilidade caótica (e.g., "Na nossa família parece que nunca nos organizamos"), Flexibilidade balanceada (e.g., "A nossa família procura novas maneiras para lidar com os problemas") e Flexibilidade rígida (e.g., "Quando se quebram as regras da família há consequências graves").

Além da FACES-IV, e de modo a obter uma compreensão mais abrangente e fidedigna do funcionamento familiar, Olson (2011) recomenda a utilização da Escala de Comunicação Familiar (do inglês "The Family Communication Scale" [FCS]; Olson \& Barnes, 2004) e da Escala de Satisfação Familiar (do inglês "Family Satisfaction Scale" [FSS]; Olson, 2004). Cada uma das escalas inclui 10 itens, como por exemplo "Na nossa família sentimo-nos satisfeitos com o modo de comunicar entre nós" (FCS) e "O tempo que passamos juntos enquanto família" (FSS).

Os itens da escala FACES-IV, assim como das escalas complementares FCS e FSS, são respondidos numa escala Likert de 5 níveis, sendo que as respostas da FACES IV expressam-se com as opções de "Discordo fortemente" até "Concordo fortemente". Já as respostas da FCS e da FSS variam de "Muito descontente" a "Muito satisfeito", sendo que quanto mais elevados os resultados nessas escalas, melhor a comunicação e maior a satisfação familiar. Os instrumentos podem ser utilizados em contexto clínico e de pesquisa. A FACES IV, FCS e FSS podem ser respondidas por membros da família acima de 12 anos. No Quadro 2, encontram-se sistematizados os artigos selecionados que apresentam pesquisas empíricas nas quais foi utilizada a FACES-IV. 
Quadro 2. Sistematização das pesquisas realizadas com a FACES-IV

\begin{tabular}{|c|c|c|c|}
\hline Referência - país & Objetivos & População / $n$ & Resultados \\
\hline $\begin{array}{l}\text { Gouveia-Pereira } \\
\text { et al. }(2020)- \\
\text { Portugal }\end{array}$ & $\begin{array}{l}\text { Validar a FACES-IV (FCS } \\
\text { + FSS), procedendo a dois } \\
\text { estudos complementares. }\end{array}$ & $\begin{array}{l}859 \text { adolescentes do } \\
\text { contexto escolar e } 67 \\
\text { adolescentes do contexto } \\
\text { hospitalar, entre os } 12 \text { e } 21 \\
\text { anos. }\end{array}$ & $\begin{array}{l}\text { Ambos os estudos resultam numa versão adaptada de } 24 \text { itens. No global, } \\
\text { a FACES-IV apresenta uma boa consistência interna }(\alpha=.81) \text {, validade } \\
\text { convergente e validade preditiva para a grande maioria das subescalas } \\
(p<.01) \text {. }\end{array}$ \\
\hline
\end{tabular}

\begin{tabular}{|c|c|c|}
\hline $\begin{array}{l}\text { Everri et al. } \\
(2020) \text { - Itália }\end{array}$ & $\begin{array}{l}\text { Validação da versão } \\
\text { italiana reduzida da } \\
\text { FACES-IV (SAD_FACES) } \\
\text { para adolescentes. }\end{array}$ & $\begin{array}{l}446 \text { estudantes } \\
\text { adolescentes entre } 14 \text { e } 16 \\
\text { anos, provenientes de } \\
\text { classe média alta, da }\end{array}$ \\
\hline
\end{tabular}

Investigar a validade transcultural dos princípios postulados pelo Modelo

Turkdogan et al. $\quad$ Circumplexo dos Sistemas (2019) - Turquia $\quad \begin{aligned} & \text { Circumplexo dos Sistem } \\ & \text { Conjugais e Familiar na }\end{aligned}$ cultura turca (com recurso à FACES-IV).

Gomes et al.

(2019) -

Portugal

Analisar as PP da FACESIV, FCS e FSS.

Analisar a relação entre o

Carvalho et al

(2018) -

Portugal

FF e as táticas de resolução de conflito na fratria (CTS2-SP, Relva et al., 2013).

Analisar as relações entre $\mathrm{FF}$, diferenciação do self e adaptabilidade de carreira de universitários.

Brasil

Martinez-

Pampliega et al.

(2017) - Espanha

Analisar as PP da versão espanhola da FACES-IV.

665 estudantes

universitários entre 18 e

41 anos.

1613 estudantes

universitários com idades

entre 17 e 51 anos.

553 portugueses entre $19 \mathrm{e}$

57 anos, estudantes e

trabalhadores.

353 estudantes entre $12 \mathrm{e}$

18 anos, estudantes do $2^{\circ} \mathrm{e}$ $3^{\circ}$ ciclo do ensino básico e secundário, na sua maioria com um irmão.

\section{0 estudantes}

universitários entre 18 e

35 anos, da região sul do Brasil.

A ESEM revelou 6 fatores, tal como a versão original da FACES-IV (validade interna). Obteve-se consistência interna satisfatória, exceto para as dimensões Emaranhamento e Caos. Verificaram-se diferenças entre rapazes e raparigas na dimensão Rigidez $(F(1,442)=4.61, p=.032, \eta$ $\left.{ }_{2}^{2}=.01\right)$, pontuando os rapazes mais elevado $(M=2.83, D P=0.86)$ do que as raparigas $(M=2.56, D P=1.01)$ (validade externa).

Os achados suportam fortemente a validade transcultural dos princípios postulados pelo Modelo: níveis balanceados de coesão $\left(\mathrm{r}_{(\mathrm{n}=1613)}=.53\right.$, $p<.01$ e flexibilidade $\left(r_{(\mathrm{n}=1613)}=.66, p<.01\right)$ são componentes-chave do FF.

Valores de consistência interna $(\alpha)$ para as dimensões da FACES-IV entre .65 e .81 . A AFC corroborou a estrutura original de 6 fatores da FACES-

IV. Observaram-se correlações positivas e significativas entre as subescalas da FACES-IV e as escalas FCS e FSS $(p<.005)$ (validade convergente). Verificou-se boa capacidade discriminante $(\Lambda=.61$, $\left.X^{2}(1)=228.32, p<.001\right)$ (validade discriminante).

A consistência interna $(\alpha)$ da FACES-IV variou entre .67 e .77. A AFC foi ajustada ao modelo de 6 fatores.

Verificaram-se associações significativas positivas médias entre a Comunicação familiar e Negociação perpetrada $(\mathrm{r}=.31 ; p \leq .01)$ e a Satisfação familiar e Vitimização da negociação $(\mathrm{r}=.30 ; p \leq .01)$ (validade de construto).

As variáveis da FACES-IV explicam 12.6\% (R2=.126) das táticas de resolução de conflito entre irmãos (validade preditiva).

Consistência interna da FACES-IV (escala completa) de $\alpha=.90$. Observouse correlação positiva do Corte emocional com todas as dimensões positivas do $\mathrm{FF}$ ( $\mathrm{r}<.30)$ : Coesão equilibrada, Flexibilidade equilibrada, Comunicação familiar e Satisfação familiar; a dimensão Coesão emaranhada se correlacionou negativamente com a fusão com os outros $(\mathrm{r}=-.30)$. Não se registaram resultados significativos entre Adaptabilidade de carreira e FF (validade de construto).

A AFC corrobora o modelo original de 6 fatores.

A consistência interna $(\alpha)$ das subescalas variou entre .63 e .84 . Correlações significativas com a FACES-II e outras medidas familiares consolidaram a validade convergente e concorrente do instrumento, enquanto a validade discriminante foi comprovada para a Coesão e Flexibilidade.

Apesar das AFE e AFC colocarem em causa o modelo de 6 fatores da FACES-IV, os autores optaram por manter a estrutura fatorial original. Consistências internas $(\alpha)$ boas a razoáveis (FACES-IV: entre .59 e .79; FCS: .90; FSS: .92). Os três instrumentos revelaram boa estabilidade temporal (de .94 a .98). Observam-se correlações elevadas entre a FCS e a FSS $(\mathrm{r}=.78, p<.001)$ (validade convergente).

Analisar as PP da FACESgregas).
584 famílias.
Koutra et al.
(2012) - Grécia Nota. AFC=análise fatorial confirmatória; $\mathrm{AFE}=$ análise fatorial exploratória; $\mathrm{CTS} 2-\mathrm{SP}=$ Conflict Tatics Scales-Sibling Version; ESEM=exploratory
structural equation modeling; FACES-II=Family Adaptability and Cohesion Evaluation Scale; FACES-IV=Family Adaptability and Cohesion Evaluation Scale; FCS=Family Communication Scale; FF=funcionamento familiar; FSS=Family Satisfaction Scale; SAD-FACES=FACES Short Version for Adolescents; $\mathrm{PP}=$ propriedades psicométricas.

\section{Estudos anteriores com a FACES-IV} apresentaram, de um modo geral, resultados positivos, ou seja, os objetivos foram alcançados validando a efetividade do instrumento para avaliar o funcionamento familiar entre adultos e adolescentes em contexto comunitário, académico e clínico. A maioria dos estudos incidiu na validação e adaptação cultural da escala, utilizando amostras de estudantes adolescentes e universitários. Houve dois temas que se diferenciaram abordando a diferenciação do self em universitários brasileiros (Fiorini \& Patta-
Badargi, 2018) e o conflito familiar expresso na relação entre irmãos na pesquisa portuguesa (Carvalho et al., 2018). Porém, e à semelhança do que acontece com o FAD, não foram encontrados estudos de validação da FACES-IV para a população brasileira.

As três pesquisas realizadas em Portugal (Carvalho et al., 2018; Gomes et al., 2019; Gouveia-Pereira et al., 2020) demonstraram as boas propriedades psicométricas do instrumento, assim como a possibilidade de sua aplicação em diferentes contextos. 
Quanto às pesquisas grega e espanhola de adaptação transcultural (Koutra et al., 2012; Martinez-Pampliega et al., 2017), cabe ressaltar que as dimensões emaranhada e rígida da coesão familiar revelaram baixas correlações, sugerindo que as mesmas não são percebidas como disfuncionais, tal como postula o Modelo Circumplexo de Sistemas Conjugal e Familiar (Olson, 2000), dado também confirmado por Cracco e Costa-Ball (2019) em pesquisa uruguaia sobre a escala de comunicação FCS (Olson \& Barnes, 2004). Além disso, a pesquisa turca de Turkdogan et al. (2019) pontua que a dimensão emaranhada da coesão familiar precisa ser revista como proposição teórica ao se examinar culturas coletivistas, porque estas enfatizam os objetivos grupais como valores cruciais para seus membros.

Destaca-se, também, a pesquisa italiana de Everri et al. (2020) que validou a versão reduzida da FACES-IV com uma amostra de adolescentes, obtendo a mesma estrutura fatorial e consistência interna encontrada no estudo de Baiocco et al. (2013), na qual os autores utilizaram a versão longa da escala (aplicada também a adolescentes). Assim, foi possível desenvolver uma medida mais compacta (com 24 itens) da FACES-IV em consonância com as transformações nas normas e estruturas familiares atuais e também com os conhecimentos sobre o funcionamento familiar a nível mundial.

Hamilton e Carr (2016), numa revisão sistemática, corroboram que a FACES-IV apresenta boas propriedades psicométricas, devendo-se proceder aos estudos de validação de forma contínua.

\section{Systemic Clinical Outcome and Routine Evaluation-15}

Stratton et al. (2010), no Reino Unido, verificaram a necessidade de criar uma medida de autorrelato de avaliação dos resultados da terapia familiar e de casal que fosse de fácil compreensão e aplicação rápida pelos membros de famílias em processo terapêutico. Assim, iniciou-se o desenvolvimento do SCORE, partindo do pressuposto sistémico de que a forma como os relacionamentos operam na família é central para o bem-estar de todos os seus membros. A medida foi desenvolvida a partir da versão de 40 itens, posteriormente evoluindo para
28 itens e, finalmente, resultando na versão composta por 15 itens.

O SCORE-15 avalia vários aspetos do funcionamento familiar que são sensíveis à mudança terapêutica como a comunicação, as dificuldades e recursos das famílias assim como também pode ser aplicado na população comunitária com objetivos de investigação (Vilaça et al., 2014).

O SCORE-15 (Stratton et al., 2014), tendo sido primeiramente desenvolvido no Reino Unido e na Irlanda, rapidamente foi alvo de validações em outros países, tais como Portugal (Vilaça et al., 2015), Suécia (Zetterqvist et al., 2019), Polônia (Jósefik et al., 2016), Itália (Paolini \& Schepisi, 2020) e a versão tailandesa na Ásia (Limsuwan \& Prachason, 2018). Os 15 itens representam descrições de vários aspetos da interação familiar e encontram-se distribuídos por três dimensões (Vilaça et al., 2015): Recursos familiares (e.g., "Somos bons a encontrar novas formas de lidar com as dificuldades"), Comunicação na família (e.g., "Na minha família muitas vezes não se diz a verdade uns com os outros") e Dificuldades familiares (e.g., "Sentimo-nos muito infelizes em nossa família").

As respostas são dadas de acordo com uma escala Likert de cinco níveis (1=Descreve-nos muito bem; 2=Descreve-nos bem; 3=Descreve-nos em parte; 4=Descreve-nos mal; e 5=Descreve-nos muito mal). Quando o objetivo é avaliar o processo terapêutico, o instrumento é completado por três perguntas qualitativas: 1) "Quais as palavras que melhor descreveriam sua família?"; 2) "Qual é o principal problema/dificuldade que o levou a recorrer à terapia?"; e 3) "Qual é o principal problema/dificuldade para sua família neste momento?". Essas respostas, além de escritas no questionário, devem ser expressas numa escala analógica de 0 a 10, sendo o significado atribuído a esta escala variável em função da questão. $\mathrm{O}$ SCORE-15 é de fácil administração, tomando 10 a 15 minutos para preenchimento individual, podendo ser respondido por adultos e adolescentes (a partir dos 12 anos). Stratton et al. (2014) sugerem a qualidade promissora do instrumento e manifestam interesse por pesquisas em populações que sofrem impacto de eventos externos, doença familiar, perda de emprego ou migração. $\mathrm{O}$ Quadro 3 apresenta as pesquisas recentes selecionadas em diferentes países europeus. 
Quadro 3. Sistematização das pesquisas realizadas com o SCORE-15

\begin{tabular}{|c|c|c|c|}
\hline Referência - país & Objetivos & População / n & Resultados \\
\hline $\begin{array}{l}\text { Paolini \& } \\
\text { Schepisi (2020) } \\
\text { - Itália }\end{array}$ & $\begin{array}{l}\text { Analisar as PP, responsividade } \\
\text { clínica e critérios normativos } \\
\text { do SCORE-15 na versão } \\
\text { italiana. }\end{array}$ & $\begin{array}{l}208 \text { famílias ( } 507 \\
\text { participantes) }\end{array}$ & $\begin{array}{l}\text { A AFC confirmou a estrutura fatorial original trifatorial, com bons } \\
\text { valores de consistência interna ( } \alpha \text { entre } .80 \text { e } .89 \text { ). Verificou-se a } \\
\text { validade discriminante, diferenciando famílias clínicas de famílias não } \\
\text { clínicas. }\end{array}$ \\
\hline
\end{tabular}

Areia et al.

(2020) -

Portugal

depressão, ansiedade, somatização e luto antecipatório complicado nos familiares de doentes oncológicos terminais.

112 participantes adultos, familiares de doentes oncológicos terminais, na sua maioria mulheres (82.1\%).

397 participantes, entre 70 famílias com filhos saudáveis e 159 famílias com filhos com problemas psiquiátricos ou comportamentais.

459 adultos portugueses estudantes e

(2018) -

Portugal

Carvalho et al. (2017) -

Compreender a relação entre as TIC's e o FF.

Investigar as PP da versão 28 15 do SCORE com base numa amostra de pacientes usuários de serviços de saúde mental que receberam tratamento multimodal.

Verificar as PP do SCORE-28 e SCORE-15 e investigar a

Hamilton et al. (2015) - Irlanda responsividade dos instrumentos na terapia familiar sistémica.
Portugal trabalhadores.

476 participantes de 157 famílias portuguesas, sendo 97 famílias com filhos adultos emergentes e 60 famílias com filhos adolescentes.

199 participantes adultos, sendo 100 pacientes ambulatoriais e 99 pacientes internados em serviços de saúde mental.

701 famílias clínicas, sendo a mãe a principal informante; 433 famílias após 3-5 meses. de depressão ( $\beta$ dep.func familiar $=.35, p<.001$ ), ansiedade ( $\beta$ ans.func familiar $=.31, p=.002)$, distress $(\beta$ dis.func familiar $=.31$, $p<.001)$ e luto antecipatório ( $\beta$ luto.func_familiar $=.34, p<.001$ ).

A AFC corroborou a estrutura fatorial tridimensional original. A estabilidade temporal (avaliada com 22 participantes de amostra não clínica) revelou uma correlação entre o $1^{\circ}$ e o $2^{\circ}$ momento de .81 para a escala total. Os valores de consistência interna $(\alpha)$ para adultos de famílias clínicas variaram entre .69 e .82., enquanto que para famílias não clínicas variou de .53 a .67. Observou-se validade convergente (correlação positiva entre o SCORE-15 e o QFD) e validade discriminante (correlação negativa entre os comportamentos pro-sociais avaliados pelo QFD e o SCORE-15)

Obteve -se uma consistência interna de $\alpha=.90$ para o SCORE-15 (escala completa). Verificou-se uma correlação positiva entre o SCORE-15 e o FDI, com escore total de $r=.40, p<.05$ (validade convergente)

Consistência interna do SCORE-15 (escala completa) de $\alpha=.90$. Correlação positiva e significativa entre o uso de TIC's e o FF (especialmente a Comunicação disruptiva: $r=.89$; Dificuldades familiares: $\mathrm{r}=90$ ) (validade de construto). O número de TIC's um fraco FF (em famílias com adolescentes) (validade preditiva).

Os resultados indicaram melhor validade fatorial para o SCORE-28, comparativamente com o SCORE-15. Obteve-se boa consistência interna para as duas escalas e respetivas subescalas $(\alpha>.70)$. Ambos os instrumentos mostraram boa validade de critério (diferenciaram entre grupos clínicos e não clínicos) e de construto (correlacionaram-se com outros construtos próximos).

Os resultados confirmaram a estrutura trifatorial para ambas as versões. Ambos instrumentos tiveram boa consistência interna (SCORE-15: $\alpha=.90$; SCORE-28: $\alpha=.93$ ) e confiabilidade no teste-reteste após 3-5 meses $(r>.70)$. Observaram-se correlações moderadas (entre -.23 e .56 ) com outras medidas de avaliação do ajustamento familiar e parental, confirmando a validade de constructo das escalas. Ambas as versões revelam capacidade discriminante entre grupos clínicos e não clínicos (tamanho do efeito elevado e valor de Cohen's d superior a .80) (validade discriminante).

Os resultados mostraram que o SCORE-15 (escala e subescalas) diferenciou significativamente $(p<.001)$ as amostras clínica e comunitária (validade discriminante). Obtiveram-se correlações moderadas ( $\mathrm{r}=-.47, p<.01)$ entre a escala e outra medida de avaliação familiar (validade convergente). Verificou-se, ainda, sensibilidade do instrumento à mudança na escala total $(\mathrm{RCI}=14 \%)$ e Comunicação familiar $(p<.05)$ (validade preditiva).
Familiares que reportem pior FF tendem a apresentar níveis elevados utilizadas e situações problemáticas explicam $18.1 \%$ da variância de
Vilaça et al

Portugal
Investigar as PP do SCORE-15 e avaliar sua sensibilidade à mudança terapêutica
618 participantes: 482 oriundos do contexto comunitário e 136 provenientes do 
após a intervenção terapêutica (Hamilton et al., 2015).

O SCORE-15 também se mostrou eficaz como instrumento complementar nas pesquisas portuguesas (Carvalho et al., 2017; Fonseca et al., 2018). Por exemplo, relacionou-se positivamente com escalas diferentes, como a Family Distress Index (FDI) na pesquisa de Fonseca et al. (2018), indicando que famílias com alto nível de stresse tendem a apresentar piores resultados de funcionamento familiar. Além disso, a pesquisa de Carvalho et al. (2017) mostrou que o maior número de tecnologias da informação facilita a comunicação familiar. A pesquisa irlandesa de O'Hanrahan et al. (2017), que utilizou o SCORE28 e SCORE-15 num contexto de serviços de saúde mental, mostrou confiabilidade e validade das versões reduzidas do SCORE com usuários internos e externos que receberam tratamento multimodal, envolvendo medicação e psicoterapia individual. Os autores consideram a utilização dos citados instrumentos para avaliar o funcionamento familiar, monitorar progresso terapêutico e avaliar resultados na terapia sistêmica com famílias de adultos com problemas mentais.

Por fim, importa realçar que não foi encontrado qualquer estudo realizado no Brasil com o SCORE-15, facto que, aliado à escassez de estudos de validação das escalas FAD e FACESIV, reforça a necessidade urgente de adaptação e validação de uma medida rápida de avaliação do funcionamento familiar para a população brasileira.

\section{Discussão}

O presente estudo incluiu uma pesquisa que se dividiu em dois momentos distintos: uma busca inicial, exploratória, focada em estudos sobre instrumentos de avaliação familiar mais utilizados no Brasil e internacionalmente; e, com base nos resultados da primeira busca, uma busca focada nos instrumentos de avaliação evidenciados na etapa anterior. Num primeiro momento, foi possível concluir que a maioria dos estudos sobre medidas de avaliação familiar foram realizados na Europa, tendo-se debruçado maioritariamente sobre a análise do FAD/GFF, FACES-IV e SCORE-15. O segundo momento, permitiu uma análise detalhada das potencialidades e fragilidades dos três instrumentos de avaliação do funcionamento familiar.

De um modo geral, o FAD/GFF, a FACES-IV e o SCORE-15 revelaram-se úteis e confiáveis em relação à mensuração das dimensões psicológicas que configuram as relações intrafamiliares, de acordo com os objetivos específicos de cada instrumento. A escala $\mathrm{FAD}$ e a sua versão reduzida GFF têm sido amplamente usadas, ao longo das últimas décadas, a nível Europeu para avaliar terapia de casal e de família (Sanderson et al., 2009), revelando-se instrumentos confiáveis e válidos utilizados em avaliação familiar (Hamilton \& Carr, 2016). As pesquisas apresentadas sobre o FAD e GFF tiveram em maior número a análise das propriedades psicométricas, efetuando validação em diferentes países. Cabe ressaltar também a utilização do FAD no contexto clínico médico como auxiliar no tratamento de doenças crónicas as quais necessitam da ajuda da família para o controle e bem-estar do paciente.

A FACES-IV teve a maioria dos estudos em validação transcultural e aplicação, relacionando o instrumento a outro constructo teórico em diferentes contextos, revelando assim que há interesse da comunidade científica em continuar utilizando a referida escala e acompanhando sua evolução em versões mais compactas como a FACES-IV para adolescente (24 itens).

O SCORE-15, por sua vez, foi desenvolvido no século XXI, como indicador de mudança terapêutica, tendo fácil aplicação e se encontrando em fase de expansão de seus estudos em vários países e continentes. Neste estudo, a maioria das pesquisas analisaram as propriedades psicométricas do instrumento, atestando a sua validação, apresentando bons resultados estatísticos, sobretudo no que diz respeito à sensibilidade do instrumento para sinalizar mudança terapêutica. O SCORE-15 tem sido referenciado pela European Family Therapy Association (Carr \& Stratton, 2017) como principal instrumento para avaliar resultados em terapia sistémica familiar e de casal. Para além disso, o SCORE-15 tem sido amplamente estudado em contexto clínico e de investigação, tanto na Península Ibérica através do acordo entre a Federação Espanhola de Associações de Terapia Familiar e a Sociedade Portuguesa de Terapia 
Familiar, como em Angola através da parceria entre a Faculdade de Psicologia e de Ciências da Educação da Universidade de Coimbra e o Instituto Politécnico Tundavala do Lubango.

De acordo com esta pesquisa, as três medidas de avaliação familiar têm a sua validade comprovada em diferentes culturas e contextos, sendo que a maioria dos estudos se focou na análise da validade de construto, confiabilidade, validade convergente, validade divergente $\mathrm{e}$ validade preditiva. Ao nível da validade de construto, a maioria dos estudos corrobora a solução fatorial original, com a exceção para a FAD visto que foram encontradas soluções fatoriais distintas da original (Barroilhet et al., 2009; Kazarian, 2010; Speranza et al., 2012) e para a FACES-IV uma vez que a estrutura fatorial é forçada num dos estudos (Koutra et al., 2012). O SCORE-15, por sua vez, tem a sua estrutura fatorial original corroborada de forma consistente nos vários estudos europeus. Globalmente, a escala GFF e o SCORE-15 são as medidas familiares que apresentam resultados estatísticos mais robustos nos vários estudos.

O FAD representa a medida de avaliação mais antiga e mais estudada, contudo, os estudos mais recentes recaem sobre a FACES-IV e o SCORE15, sugerindo assim um crescente interesse científico por estas duas medidas. Por fim, o SCORE-15 destaca-se positivamente pelo facto de ser uma medida contemporânea breve e, por isso, de maior facilidade na administração em contexto clínico e comunitário.

Apesar de estas três medidas serem amplamente estudadas e aplicadas a nível Europeu, até à data, apenas o FAD e a sua escala GFF estão validadas para o contexto brasileiro. De acordo com Fiorini e Patta-Badargi (2018), encontra-se a decorrer o processo de validação da FACES-IV para o Brasil, não estando ainda asseguradas as suas competências psicométricas neste contexto. Em relação ao SCORE-15, a medida familiar mais recente, este instrumento não foi ainda objeto de estudo de validação para a população brasileira. Foi ainda possível constatar que, dos 25 estudos selecionados, apenas três foram realizados no Brasil (dois estudos sobre o FAD/GFF e um estudo sobre a FACES-IV), o que, por um lado reflete a importância atribuída às produções científicas focadas na avaliação familiar na Europa e, por outro, evidencia o atraso do seu estudo no Brasil.

O estudo apresentado propôs-se contribuir para o enriquecimento dos conhecimentos que compõem a ciência da família. Em particular, um dos principais contributos deste estudo consiste no levantamento e apreciação dos instrumentos de avaliação familiar mais utilizados na Europa e Brasil. Além disso, ao evidenciar a carência de estudos de validação de medidas familiares no Brasil e, consequentemente, a escassez de achados empíricos sobre o funcionamento familiar brasileiro, este trabalho aponta caminhos para a investigação futura.

Como limitações deste estudo, aponta-se o foco em pesquisas realizadas no Brasil e Europa. A opção de delimitar a nossa análise a pesquisas europeias, após se constatar que a grande maioria dos estudos sobre avaliação familiar foram desenvolvidas neste território, permitiu uma análise focada desta temática, tendo contudo, excluído trabalhos oriundos de outros territórios. Neste sentido, a consideração exclusiva de pesquisas sobre o FAD/GFF, a FACES-IV e o SCORE-15 representa igualmente uma limitação. A busca de artigos, de carácter exploratório, levada a cabo num primeiro momento pelos autores para identificação dos instrumentos mais utilizados constitui uma fragilidade deste estudo, uma vez que decorreu de forma menos criteriosa (e.g., não foi controlado o número de artigos identificados, selecionados e elegíveis). Por fim, o facto de terem sido considerados apenas artigos publicados em inglês, português e espanhol também limitou o alcance deste estudo.

Para além dos achados científicos necessários, torna-se fundamental contribuir para o desenvolvimento de pesquisas transculturais que podem promover trocas enriquecedoras entre as nações. Como sugestão de continuidade do trabalho realizado, sugere-se o levantamento e avaliação da qualidade psicométrica das medidas de avaliação familiar mais utilizadas em todo o mundo. Especificamente no Brasil, ressalta-se a importância da continuidade da validação dessas medidas, considerando sua imensa diversidade demográfica, social e cultural. Atendendo aos resultados promissores dos estudos europeus focados no SCORE-15, a validação deste instrumento para o Brasil faz-se necessária. 


\section{Referências}

Almeida, A. C., Leandro, M. E., \& Pereira, M. G. (2020). Psychometric properties of the Portuguese adaptation of General Functioning of Family Assessment Device: A comparative study. Families, Systems, \& Health, 38(2), 162-171. https://doi.org/10.1037/fsh0000471

Areia, N., Major, S., Fonseca, G., Oliveira, V., \& Relvas, A. P. (2020). Prevalência e preditores de morbilidade psicológica nas famílias de doentes oncológicos terminais. Psicologia, Saúde \& Doenças 2(1), 169-175. http://dx.doi.org/10.15309/20psd210125

Baiocco, R., Cacioppo, M., Laghi, F., \& Tafà, M. (2013). Factorial and construct validity of FACES IV among Italian adolescents. Journal of Child and Family Studies, 22(7), 962-970. https://doi.org/10.1007/s10826-012-9658-1

Ballash, N. G., Pemble, M. K., Usui, W. M., Buckley, A. F., \& Woodruff-Borden, J. (2006). Family functioning, perceived control, and anxiety: A mediational model. Journal of Anxiety Disorders, 20(4), 486-497. https://doi.org/10.1016/j.janxdis.2005.05.002

Banovcinova, A., \& Levicka, K. (2015). The impact of the financial income on the family communication. Revista Romaneasca Pentru Educatie Multidimensionala, 7(2), 35-46. https://ideas.repec.org/a/lum/rev1rl/v7y2015i2 p35-46.html

Barroilhet, S., Cano-Prous, A., Cervera-Enguix, S., Forjaz, M. J., \& Guillén-Grima, F. (2009). A Spanish version of the family assessment device. Social Psychiatry and Psychiatric Epidemiology, 44(12), 1051-1065. https://doi.org/10.1007/s00127-009-0022-8

Bray, J. H. (1995). Family assessment: Current issues in evaluating families. Family Relations: An Interdisciplinary Journal of Applied Family Studies, 44(4), 469-477. https://doi.org/10.2307/585001

Carr, A. (2009a). The effectiveness of family therapy and systemic interventions for adultfocused problems. Journal of Family Therapy, 31(1), 46-74.

https://doi.org/10.1111/j.1467-

6427.2008.00452.x

Carr, A. (2009b). The effectiveness of family therapy and systemic interventions for child- focused problems. Journal of family therapy, 31(1), 3-45.

https://doi.org/10.1111/j.1467-

6427.2008.00451.x

Carr, A., \& Stratton, P. (2017). The Score family assessment questionnaire: A decade of progress. Family Process, 56(2), 285-301. https://doi.org/10.1111/famp.12280

Carvalho, J. L., Francisco, R., \& Relvas A. P. (2017). ICTS and family functioning: A study on Portuguese families with adolescents and emerging adults. Contemporary Family Therapy, 39, 281-288.

https://doi.org/10.1007/s10591-017-9436-8

Carvalho, J. L., Relva, I. C., \& Fernandes, O. M. (2018). Funcionamento familiar e estratégias de resolução de conflitos na fratria. Análise Psicológica, 36(1), 61-73.

https://doi.org/10.14417/ap.1354

Conselho Federal de Psicologia (n.d.). Sistema de Avaliação de Testes Psicológicos. https://satepsi.cfp.org.br/

Cracco, C., \& Costa-Ball, C. D. (2019). Propriedades psicométricas de la Escala de Comunicación Familiar. Revista Iberoamericana de diagnóstico y Evaluación - $e$ Avaliação Psicológica, 51(2), 77-86. https://doi.org10.21865/RIDEP51.2.06

Dessen, M. A. (2010). Estudando a família em desenvolvimento: Desafios conceituais e teóricos. Psicologia: Ciência e Profissão, 30, 202-219.

https://doi.org/10.1590/S141498932010000500010

Epstein, N. B., Baldwin, L. M., \& Bishop, D. S. (1983). The McMaster assessment device. Journal Family Therapy, 9(2), 171-80. https://doi.org/10.1111/j.17520606.1983.tb01497.x

Everri, M., Caricati, L., Mancini, T., Messena, M., \& Fruggen, L. (2020). Italian validation of family adaptability and cohesion scale (FACES IV) short version for adolescents: SAD_FACES. Journal of Child and Families Studies, 29, 2507-2514. https://doi.org/10.1007/s10826-020-01771-9

Ferriolli, S. H. T., Marturano, E. M., \& Puntel, L. P. (2007). Contexto familiar e problemas de saúde mental infantil no Programa Saúde da Família. Revista de Saúde Pública,41(2), 
251-259.https://doi.org/10.1590/S003489102006005000017

Fiorini, M. C., \& Patta-Bardagi, M. (2018). Funcionamento familiar, diferenciação do self e adaptabilidade de carreira de universitários brasileiros. Psicologia desde el Caribe, 35(3), 210-223. https://doi.org/10.14482/psdc.35.3.155.2

Fonseca, G., Crespo, C., McCubbin, L., Areia, N., \& Relvas, A. P. (2018). Psychometric study of the Portuguese version of the family distress (FDI). Family, Systems and Health, 36(2), 159-168. https://doi.org/10.1037/fsh0000342

García-Méndez, M., Rivera-Aragón, S., ReyesLagunes, I., \& Díaz-Loving, R. (2006). Construcción de una escala de funcionamiento familiar. Revista Iberoamericana de Diagnóstico y Evaluación - e Avaliação Psicológica, 2(22), 91-110. https://doi.org/10.33881/2027-1786.rip.10103

Gomes, H. M. S., Peixoto, F., \& Gouveia-Pereira, M. (2019). Portuguese validation of Family Adaptability and Cohesion Evaluation Scale FACES IV. Journal of Family Studies, 25(4), 447-494.

https://doi.org/10.1080/13229400.2017.13861 21

Gouveia-Pereira, M., Gomes, H., Miranda, M., \& Candeias, M. J. (2020). Coesão e flexibilidade familiar: Validação do pacote FACES IV junto de adolescentes portugueses. Análise Psicológica, 1(38), 111-126.

http://dx.doi.org/10.14417/ap.1651

Hamilton, E., Carr, A., Cahill, P., Cassels, C., \& Hartnett, D. (2015). Psychometric and responsiveness to change of 15 and 28 -item versions of the SCORE: A family assessment questionnaire. Family Process, 54(3), 454463. https://doi.org/10.1111/famp.12117

Hamilton, E., \& Carr, A. (2016). Systematic review of self-report family assessment Measures. Family Process, 55(1), 16-30. https://doi.org/10.1111/famp.12200

Jósefik, B., Matusiak, F., Wolska, M., \& Ulasinska, R. (2016). Family therapy processworks on the polish version of SCORE-15 tool. Psychiatria Polska, 50(3), 607-619. https://doi.org/10.12740/pp/onlinefirst/42894

Kazarian, S. S. (2010). Culture appropriateness of the family assessment device (FAD) in the case of ethnic Armenian adolescents in Lebanon. International Journal of Social Psychiatry, 56(3), 230-238. https://doi.org/10.1177/0020764009105646

Koutra, K., Triliva, S., Roumeliotaki, T., Lionis, C., \& Vgontzas, A. N. (2012). Cross-cultural adaptation and validation of the Greek version of the family adaptability and cohesion evaluation scales IV package (FACESIV package). Journal of Family Issues, 34(2), 1647-1672.

https://doi.org/10.1177\%2F0192513X124628 18

Lebow, J., \& Stroud, C. B. (2016). Avaliação do funcionamento conjugal e familiar: Modelos e instrumentos predominantes. In F. Walsh (Ed.). Processos normativos da família: diversidade e complexidade ( $4^{\mathrm{a}}$ ed., pp 501528). Artmed.

Limsuwan, N., \& Prachason, T. (2018). The reliability and validity of the 15-item Systemic Clinical Outcome and Routine Evaluation (SCORE-15). Thai version (SCORE-15). Journal of Family Therapy 42(1), 119-128.

https://doi.org/10.1111/1467-6427.12248

Martinez-Pampliega, A., Merino, L., Iriarte, L., \& Olson, D. H. (2017). Psychometric properties of the spanish version of the Family Adaptability and Cohesion Evaluation Scale IV. Psicothema, 29(3), 414-420. https://doi.org/10.7334/psicothema2016.21

Miller, I. W., Ryan, C. E., Keitner, G. I., Bishop, D. S., \& Epstein, N. B. (2000). The McMaster approach to families: Theory, assessment, treatment and research. Journal of Family Therapy, 22(2), 168-189. https://doi.org/10.1111/1467-6427.00145

Ministério da Saúde (2013). Cadernos de atenção básica: Saúde mental. Editora MS. http://189.28.128.100/dab/docs/portaldab/publ icacoes/caderno_34.pdf

Minuchin, S. (1982). Famílias: Funcionamento e Tratamento. Edições Artes Médicas.

O'Hanrahan, K., Daly White, M., Carr, A., Cahill, P., Keenleyside, M., Fitzhenry, M., Harte, E., Hayes, J., Noonan, H., O'Shea, H., McCullagh, A., McGuinness, S., Rodgers, C., Whelan, N., Sheppard, N. and Browne, S. (2017). Validation of 28 and 15 items versions 
of the SCORE: Family assessment questionnaire with adult mental health service users. Journal of Family Therapy, 39(1), 4-20. https://doi.org/10.1111/1467-6427.12107

Olson, D. H. (2000). Marital and family systems. Journal of Family Therapy, 22(2), 144-167. https://doi.org/10.1111/1467-6427.00144

Olson, D. H. (2004). Family Satisfaction Scale (FSS). Life Innovations.

Olson, D. H. (2011). FACES IV and the circumplex model: Validation study. Journal of Marital and Family Therapy, 37(1), 64-80. https://doi.org/10.1111/j.17520606.2009.00175.x

Olson, D. H., \& Barnes, H. (2004). Family Communication Scale (FCS). Life Innovations.

Olson, D. H., \& Gorall, D. M. (2003). Circumplex model of marital \& family systems. In F. Walsh (Ed.). Normal family processes: Growing diversity and complexity (p. 514548). The Guilford Press.

https://psycnet.apa.org/doi/10.4324/97802034 28436_chapter_19

Olson, D. H., Gorall, D. M., \& Tiesel, J. W. (2007). FACES IV \& the Circumplex Model: Validation study.

https://citeseerx.ist.psu.edu/viewdoc/downloa $\mathrm{d}$ ?doi=10.1.1.582.1200\&rep=rep1\&type=pdf

Olson, D. H., Sprenkle, D. H., \& Russell, C. (1979). Circumplex model of marital and family systems: I cohesion and adaptability dimensions, family types and clinical implications. Family Process, 18(1), 3-28. https://doi.org/10.1111/j.1545-

5300.1979.00003.x

Padilla, A. M. (2002). Hispanic psychology: A 25-year retrospective look. Online Readings in Psychology and Culture, 3(19). https://doi.org/10.9707/2307-0919.1025

Paolini, D., \& Schepisi, L. (2020). The Italian version of SCORE-15: Validation and potential use. Family Process, 59(4), 17891800. https://doi.org/10.1111/famp.12495

Pavarini, S. C., Bergola, A. G., Luchesi, B. M., Oliveira, D., Orlandi, F. S., Moura, F. G., Silva, H., R. O., Oliveira, N. A., Zazzetta, M. S., Santos-Orlandi, A. A., \& Ottaviani, A. C. (2020). Social and health-related predictors of family function in older spousal caregivers: a cross-sectional study. Dementia \& Neuropsychologia, 14(4), 372-378. https://doi.org/10.1590/1980-57642020dn14040007

Pereira-Silva, N. L., Dessen, M. A., \& Barbosa, A. J. G. (2015). Ajustamento conjugal: comparação entre casais com e sem filhos com deficiência intelectual. Psico-USF, 20(2), 297-308.

http://dx.doi.org/10.1590/1413-

82712015200210

Pires, T., Avanci, J. Q., \& Pesce, R. P. (2016). Cross-cultural adaptation of the general functioning scale of the family. Revista de Saúde Pública, 50(32), 1-11. https://doi.org/10.1590/S15188787.2016050005832

Relva, I., Fernandes, O. M., \& Costa, R. (2013). Psychometric properties of the revised conflict tactics scales: Portuguese sibling version (CTS2-SP). Journal of Family Violence, 28(5), 46-62. https://doi.org/10.1007/s10896-013-9530-0

Resolução n. 9, de 25 de abril de 2018. Estabelece diretrizes para a realização de Avaliação Psicológica no exercício profissional da psicóloga e do psicólogo, regulamenta o Sistema de Avaliação de Testes Psicológicos SATEPSI e revoga as Resoluções $\mathrm{n}^{\circ}$ 002/2003, $n^{\circ} 006 / 2004$ e $n^{\circ} 005 / 2012$ e Notas Técnicas $n^{\circ}$ 01/2017 e 02/2017. Brasília: Conselho Federal de Psicologia. http://www.crp11.org.br/upload/Resolu\%C3\% A7\%C3\%A3o-CFP-n\%C2\%BA-09-2018com-anexo.pdf

Sanderson, J., Kosutic, I., Garcia, M., Melendez, T., Donoghlue, J., Perumbilly, S., Franzen, C., $\&$ Anderson, S. (2009). The measurement of outcome variables in couples and family therapy research. Journal of Family Therapy, 37(3), 239-257.

https://doi.org/10.1080/01926180802405935

Souza, J., Abade, F., Silva, P. M. C., \& Furtado, E. F. (2011). Avaliação do funcionamento familiar no contexto da saúde mental. Revista de Psiquiatria Clínica, 38(6), 254-259. https://doi.org/10.1590/S010160832011000600007

Speranza, M., Guénolé, F., Revah-levy, A., Egler, P. J., Negadi, F., Falissard, B., \& Baileyte, J. 
M. (2012). The French version of the family assessment device. Canadiam Journal of Psychiatry, 57(9), 570-577. https://doi.org/10.1177/070674371205700908

Staccini, L., Tomba, E., Grandi, S., \& Keitner, G. I. (2015). The Evaluation of family functioning by the family assessment device: A systematic review of studies in adult clinical population. Journal Family Process, 54(1), 94-115.

https://doi.org/10.1111/famp.12098

Stratton, P., Bland, J., Janes, E., \& Lask, J. (2010). Developing an indicator of family function and a practicable outcome measure for systemic family and couple therapy: The SCORE. Journal of Family Therapy, 32(3), 232-258.

https://doi.org/10.1111/j.14676427.2010.00507.x

Stratton, P., Lask, J., Bland, J., Nowotry, E., Evans, C., Singh, R., Janes, E., \& Peppiant, A. (2014). Detecting therapeutic improvement early in therapy: Validation of the SCORE-15 index of family functioning and change. Journal of Family Therapy, 36, 3-19. https://doi.org/10.1111/1467-6427.12022

Teodoro, M. L.M. (2012). Alguns instrumentos para avaliação familiar no Brasil. In M. N. Baptista, \& M. L. M. Teodoro (Orgs.). Psicologia da família: Teoria, avaliação e intervenções (pp 168-175). Edições Artes Médicas.

Timmerby, N., Cosci, F., Watson, M., Csillag, C., Schimitt, F., Stek, B., Bech, P., \& Thastum, M. (2018). A Confirmative clinimetric analysis of the 36- items family assessment device. Nordic Journal of Psychiatry, 72(4), 268-272.

https://doi.org/10.1080/08039488.2018.1435721

Traebert, E., Santos, K., Carvalho, L. M., Silva, J., \& Traebert, J. (2016). Preliminary stages of cross-cultural adaptation of the Brazilian Portuguese version of McMaster Family Assessment Device (FAD). Jornal Brasileiro de Psiquiatria, 65(3), 201-208. https://doi.org/10.1590/0047-2085000000125

Turkdogan, T., Duru, E., \& Balkis, M. (2019). Circumplex modal of family functioning in Turkish culture: Western family systems modal in a Eurasian country. Journal of comparative Family Studies, 50(2), 183-199. https://doi.org/10.3138/jcfs.50.2.005

Van Schoors, M., Caes, L., Knoble, N. B., Goubert, L., Verhofstadt, L. L., \& Alderfer, M. A. (2016). Systematic review: Associations between family functioning and child adjustment after pediatric cancer diagnosis: A meta-analysis. Journal of Pediatric Psychology, 42(1), 6-18. https://doi.org/10.1093/jpepsy/jsw070

Vilaça, M., Silva, J., \& Relvas, A. P. (2014). Systemic Clinical Outcome Routine Evaluation (SCORE-15). In A. P. Relvas, \& S. Major (Coords.). Instrumentos de Avaliação Familiar: Funcionamento e Intervenção (Vol. 1, pp. 23-41). Imprensa da Universidade de Coimbra. https://dx.doi.org/10.14195/978-989-26-0839-6

Vilaça, M., Sousa, B., Stratton, P., \& Relvas, A. P. (2015). The 15-item Systemic Clinical Outcome and Routine Evaluation (SCORE15) scale: Portuguese validation studies. The Spanish Journal of Psychology, 18(87), 1-10. https://doi.org/10.1017/sjp.2015.95

Walsh, F. (2016). Processos normativos da família: Diversidade e complexidade. ( ${ }^{\mathrm{a}} \mathrm{ed}$.). Edições Artes Médicas.

Zetterqvist, M., Emeroth Hannel, H., Wadsby, M., Cocozza, M., \& Gustafsson, P. A. (2019). Validation of the Systemic Clinical Outcome and Routine Evaluation (SCORE 15) selfreport questionnaire: Index of family functioning and change in Swedish families. Journal of Family Therapy, 42(1), 129-148. https://doi.org/10.1111/1467-6427.12255 